\title{
Resultados y aprendizajes de la evaluación de políticas agropecuarias en Uruguay
}

\author{
Mainstreaming evaluation in public policies in Uruguay. \\ Results and lessons learned in agriculture policies
}

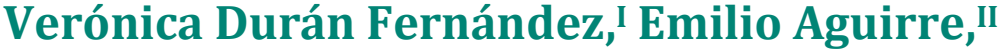 Juan Baraldo,, Elisa Hernández, ${ }^{\text {IV }}$ Hugo Lagunav}

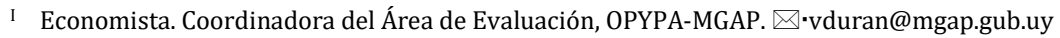 \\ https://orcid.org/0000-0002-2934-6027 \\ II Economista. Especialista en evaluación en OPYPA-MGAP. $\ \cdot$ emaguirre@mgap.gub.uy \\ https://orcid.org/0000-0002-6004-1918 \\ III Economista. Especialista en evaluación en OPYPA-MGAP. \·jbaraldo@mgap.gub.uy \\ https://orcid.org/0000-0002-6855-4670

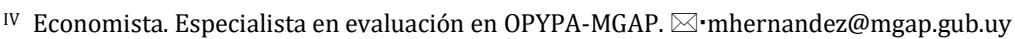 \\ https://orcid.org/0000-0001-8604-8504 \\ V Economista. Especialista en evaluación en OPYPA-MGAP. $₫ \cdot$ hlaguna@mgap.gub.uy \\ https://orcid.org/0000-0003-3644-1046
}

RECIBIDO: 11.9 .2020

ACEPTAD0: 5.11.2020

\section{Resumen}

El Ministerio de Ganadería, Agricultura y Pesca (MGAP) se propuso destinar esfuerzos al fortalecimiento de la cultura de evaluación de sus políticas agropecuarias, orientadas al aprendizaje institucional y mejora de la toma de decisiones para el logro de sus objetivos institucionales. Para ello se conformó, dentro de la Oficina de Programación y Política Agropecuaria (OPYPA), un grupo técnico especializado —el Área de Evaluación de Políticas Agropecuarias- y se desarrolló un sistema de información. Así, fue posible realizar con altos estándares de calidad evaluaciones de diversas intervenciones, que combinaron técnicas econométricas de evaluación de impacto con métodos cualitativos y análisis teóricos. Este artículo sintetiza los principales resultados y lecciones 
aprendidas en dicho proceso, en tres partes. En la primera se resumen las principales razones y fundamentos para la evaluación de políticas en el MGAP y el diseño institucional elegido. En segundo lugar se comentan los avances en la institucionalización de la evaluación de políticas dentro del MGAP. Finalmente, se presentan los principales productos realizados y las lecciones aprendidas para el uso de las evaluaciones en la toma de decisiones basadas en evidencia.

Palabras clave: política agraria, evaluación, evaluación de impacto, desarrollo rural, Uruguay.

\section{Abstract}

With the goal of improving public policy design and to better achieve institutional goals, the Ministry of Livestock, Agriculture and Fisheries (MGAP) of Uruguay allocated resources towards strengthening the evaluation of its agricultural policies. To this end, the Agricultural Policy Evaluation Area was created within the Office of the Agricultural Programming and Policy Office (OPYPA). This multidisciplinary team is specialized in impact evaluations of public interventions combining econometric techniques with qualitative methods and theoretical analysis. This document presents the main results and lessons learned in this process, in three sections. The first section summarizes the goals for the impact evaluation of MGAP policies and the basis for the institutional arrangement. Then, the progress made in mainstreaming policy evaluation within MGAP is discussed. Finally, the main results and the lessons learned from the application of evaluations in evidence-based decision making are presented.

Keywords: agricultural policy, evaluation, rural development, Uruguay.

\section{Fundamentos para la evaluación de políticas agropecuarias y del diseño institucional en el MGAP}

El fin último de la evaluación, como una instancia más del ciclo de políticas públicas, es la mejora de las intervenciones en su diseño, implementación y resultados. Las evaluaciones permiten asimismo rendir cuentas sobre los resultados de las políticas y los proyectos, y ser transparentes con los beneficiarios y la ciudadanía en general.

Para las autoridades ministeriales es de gran interés disponer de evidencia robusta sobre en qué medida las intervenciones logran los propósitos o impactos buscados, ya que ello está muy vinculado con la utilidad y pertinencia de las diferentes herramientas de política para alcanzar los objetivos estratégicos (Durán et. al, 2019). 
Aunque el Ministerio de Ganadería, Agricultura y Pesca (MGAP) tiene una larga experiencia acumulada en el monitoreo y seguimiento de programas y proyectos, no son muchas las políticas que han sido evaluadas desde el punto de vista del logro de sus objetivos propiamente dichos. Por lo tanto, es poco lo que se conoce acerca de los efectos sobre la población beneficiaria atribuibles a la política agropecuaria, así como de la conveniencia económica, incluidos los impactos ambientales, de las herramientas de política.

La Oficina de Programación y Política Agropecuaria (OPYPA) del MGAP es una oficina técnica cuya misión es asesorar a las autoridades ministeriales en el diseño, implementación y evaluación de políticas públicas para el desarrollo sostenible del sector agropecuario. En 2014, la OPYPA propuso construir y fortalecer capacidades internas, a través de la conformación de un equipo técnico especializado, con la misión de asesorar a las autoridades ministeriales sobre los resultados, consecuencias e impactos de las herramientas de política agropecuaria (Durán et al., 2014). Este diseño institucional permite aprovechar un conjunto de fortalezas y oportunidades, aunque no está exento del desafío de mitigar algunos riesgos y desventajas.

Las evaluaciones son desarrolladas fuera de las unidades que ejecutan e implementan las herramientas de política, por lo que, si bien son internas al MGAP, no constituyen autoevaluaciones.

Entre las ventajas de este diseño institucional se destacan la construcción de una cultura de evaluación y el aprovechamiento de los conocimientos de técnicos y expertos del MGAP. Esto último es fundamental para comprender profundamente la teoría del cambio y contextualizar adecuadamente las intervenciones, requisitos fundamentales para lograr buenas evaluaciones. Además, es posible que las recomendaciones de mejora de las políticas y los reportes de evaluación sean mejor aceptados si son realizados desde dentro del MGAP.

Sin embargo, el diseño plantea el desafío de mitigar el riesgo de autocomplacencia institucional. Para ello, el Área de Evaluación ha incorporado socios estratégicos externos, que fortalecen la imagen y dan garantías técnicas acerca de la independencia de la evaluación (una mirada externa): la Dirección de Gestión y Evaluación del Estado (AGEV) de la Oficina de Planeamiento y Presupuesto (OPP) y el Banco Interamericano de Desarrollo (BID). Además, el equipo busca usualmente la revisión entre pares dando a conocer los resultados de las evaluaciones en seminarios académicos, y está en camino a presentar los informes de evaluación en revistas arbitradas.

Pueden aplicarse diferentes metodologías de evaluación para responder a las preguntas de política relevantes, con base en la evidencia empírica y la teoría económica. La metodología utilizable en cada caso depende, por un lado, de la etapa del ciclo de políticas en que se encuentre la intervención (antes, durante o después de la implementación) y, por otro, del propósito de la evaluación o tipo de pregunta que se quiere 
responder (HM Treasury, 2020; Departamento Nacional de la Planeación, 2012). De este modo, en las evaluaciones ex ante se podrán aplicar metodologías cualitativas como el árbol de problemas, la teoría del cambio y evaluaciones lógicas y de diseño, con el objetivo de identificar el problema público que se quiere resolver y conocer sus causas, así como evaluar la formulación y el diseño lógico de la intervención. Entre las metodologías cuantitativas que se pueden aplicar ex ante se destacan el análisis costo-beneficio y los modelos de equilibrio general computables, con el propósito de evaluar si la intervención sería conveniente desde el punto de vista social o de la economía en su conjunto, y analizar si la política tendría efectos macroeconómicos o sobre otros sectores de actividad o de la población, entre otros.

Cuando la evaluación se realiza ex post, y si las circunstancias lo permiten, es posible responder en qué medida los beneficiarios lograron mejorar su situación gracias al programa o si la intervención produjo impactos diferenciales sobre subpoblaciones de beneficiarios mediante las técnicas econométricas de evaluación de impacto. A modo de ejemplo, el Área de Evaluación ha utilizado la técnica del árbol de problemas para comprender las causas del problema público que se quiere solucionar con intervenciones como las de desarrollo rural, entre otras. La teoría del cambio y las evaluaciones lógicas y de diseño apuntan a conocer cómo cada medida de política contribuye a mitigar alguna de las causas del problema, al tiempo que ayudan a mejorar el diseño de las intervenciones. Los análisis costo-beneficio desarrollados por el Área de Evaluación han permitido conocer si las medidas de política analizadas son convenientes desde el punto de vista de la economía en su conjunto y para determinados actores, así como incorporar algunos impactos ambientales.

También tiene interés para la toma de decisiones advertir si la medida de política analizada tiene efectos no buscados, por ejemplo, sobre otros sectores de actividad distintos al agropecuario, o sobre determinados sectores de la población. Los impactos macroeconómicos - sobre el PIB, el comercio exterior, el tipo de cambio real, los salarios, las cuentas públicas, etc.- son por demás importantes cuando se analizan políticas transversales que generan efectos inducidos o de segunda vuelta. Para intentar responder estas últimas preguntas, el Área de Evaluación ha implementado un modelo de equilibrio general computable. Por su parte, para el análisis de proyectos dirigidos a una población focalizada, las técnicas de evaluación de impacto han permitido conocer en qué medida la intervención logró los efectos deseados, así como identificar si las mejoras en los beneficiarios pueden atribuirse al programa. También es de gran utilidad indagar sobre los posibles impactos diferenciales que pueda tener la intervención sobre distintas subpoblaciones y sobre indicadores ambientales específicos, como las emisiones de gases de efecto invernadero o la calidad del agua, aspectos que el Área de Evaluación está incorporando en sus más recientes estudios. 


\section{Avances en institucionalización de la evaluación de políticas agropecuarias en el MGAP}

Desde los inicios, el Área de Evaluación de Políticas Agropecuarias se ha planteado afianzar la cultura de evaluación en el MGAP (Durán et. al, 2019). Entre los hitos más notorios en la institucionalización de la evaluación caben señalar:

1. Equipo técnico. Se conformó un equipo de especialistas en métodos de evaluación, que trabaja dentro de OPYPA en forma coordinada y complementaria con diversas áreas y técnicos del Ministerio. Asimismo, se desarrolla un proceso de formación y capacitación permanente que incluye intercambio con especialistas internacionales y regionales y con expertos y la academia nacional.

2. Desarrollo de un sistema de información único adaptado a las necesidades de las evaluaciones y con protección de la confidencialidad de los datos. Desde 2016 se trabajó fuertemente en la construcción de un sistema informático que unifica y da consistencia a la información proveniente de distintas fuentes: el Censo Agropecuario de 2011, encuestas propias especialmente realizadas para las evaluaciones y diversos registros administrativos.

3. Definición de un protocolo de evaluación en el MGAP. Se elaboró una guía que orienta los pasos a seguir para el adecuado desarrollo de las evaluaciones (Durán y Hernández, 2019). Ello incluye la participación de un grupo interinstitucional e interdisciplinario que acompaña y valida cada uno de los hitos de la evaluación: desde la definición del alcance de la evaluación, la teoría del cambio de la intervención e indicadores a evaluar, la selección de la metodología acorde a los datos disponibles, el proceso de análisis y la difusión de los resultados.

4. Fortalecimiento de capacidades en herramientas de evaluación diversas. El Área de Evaluación ha trabajado en el desarrollo de una caja de herramientas metodológicas acorde a las necesidades en el MGAP (Durán et al., 2016). Si bien se han aplicado técnicas cualitativas, la mayoría de las evaluaciones se han implementado con metodologías cuantitativas basadas en métodos econométricos.

5. Articulación con actores del sistema. Para propiciar el continuo fortalecimiento de capacidades y lograr una adecuada calidad de las evaluaciones es clave lograr retroalimentación en instancias de intercambio con colegas, académicos y otros actores. Para ello, el Área de Evaluación ha organizado seminarios, cursos y talleres donde se debaten tópicos de interés con invitados tanto locales como externos. En la página web del área (https://www.gub.uy/ministerio-ganaderia-agricultura-pesca/politicas-y-gestion/seminarios-sobre-topicos-evaluacionpoliticas) están disponibles las presentaciones realizadas en distintos 
seminarios y talleres. Además, OPYPA y AGEV firmaron un convenio de trabajo para el desarrollo conjunto de evaluaciones.

\section{Principales evaluaciones y lecciones aprendidas}

En esta sección se presentan brevemente los principales estudios desarrollados por el Área de Evaluación de Políticas Agropecuarias, así como el uso que se les ha dado para la mejora en la toma de decisiones de política.

Las intervenciones evaluadas han sido seleccionadas y priorizadas con base en los lineamientos de las autoridades ministeriales y difieren en la etapa del ciclo de políticas en que se encuentran. Al tiempo que algunos de los estudios ya han finalizado, otros se encuentran en pleno desarrollo.

Cabe resaltar que la agenda de evaluaciones en el MGAP incorpora las prioridades e impronta de cada administración de gobierno, pero también se desarrolla gracias a una planificación técnica con visión de largo plazo que permite ir más allá de los límites del ciclo político y desarrollar investigaciones que requieren varios años de estudio y la asignación presupuestal de más de un gobierno.

\section{Evaluaciones de impacto}

Una herramienta de política usual a nivel internacional para fomentar el desarrollo agropecuario son los programas focalizados de transferencia tecnológica, basados en la provisión de asistencia técnica y subsidios a los productores. El MGAP, a través de su Dirección General de Desarrollo Rural, implementó diversos proyectos con el objetivo de solucionar dos problemas de largo plazo. Primero, la baja tasa de adopción de tecnologías disponibles, en especial, por los productores ganaderos familiares, lo que resulta, en primer lugar, en una productividad por debajo de su potencial, con baja tasa de crecimiento y amplias brechas entre productores, además de una gran vulnerabilidad ante la variabilidad climática; en segundo lugar, los recursos naturales de base del sector agropecuario que resultan amenazados (erosión del suelo y del campo natural y contaminación de fuentes de agua, entre otros) (Aguirre et al., 2017).

Aunque en la última década las evaluaciones de impacto de políticas agropecuarias con metodologías basadas en la construcción de un grupo contrafactual tuvieron un impulso a nivel internacional, todavía es escasa la evidencia empírica sobre el impacto de este tipo de intervenciones (Independent Evaluation Group [IEG], 2011; López et al., 2017; De Janvry et al., 2017). 
Uruguay fue el primer país que implementó evaluaciones sobre el efecto causal de programas focalizados dirigidos a la ganadería (López y Maffioli, 2008; Mullally y Maffioli, 2015; Aguirre, Baraldo, Durán, Gesto et al., 2017; Durán et al., 2018). Esto se explica, posiblemente, por el perfil productivo del país, así como por la disponibilidad de información (sistema de trazabilidad del ganado, obligatorio y de cobertura nacional).

Así, el Área de Evaluación de Políticas, en cooperación con AGEV, estudió los impactos del Programa Agua para la Producción Animal (APA) que se ejecutó entre 2011 y 2013 (Aguirre, Baraldo, Durán, Gesto et al., 2017). La intervención consistió en otorgar subsidios parciales para que los productores lograran disponer de agua en las parcelas donde pastorean los animales. Según la teoría del cambio, se esperaba que las soluciones de agua aumentaran la resiliencia de estos productores ante eventos de sequía, al tiempo que mejoraran su productividad e ingresos. Se halló evidencia de que el cofinanciamiento de inversiones y asistencia técnica provistos por la intervención produjeron impactos positivos sobre la producción y la productividad en la subpoblación de productores lecheros. Además, una evaluación costo-beneficio ex post permitió conocer que las inversiones del proyecto fueron rentables económicamente, con un período de repago de las inversiones de aproximadamente cinco años. Este hallazgo, unido a que la mayor parte de los beneficios son apropiables por los productores tamberos, pone en duda si el diseño más adecuado de la política es un subsidio - como el otorgado por APA y buena parte de las intervenciones de la DGDR - u otros que faciliten el financiamiento como créditos de largo plazo. Por otra parte, se encuentra en proceso una evaluación para la subpoblación de ganaderos de carne.

También se evaluaron las principales convocatorias del Programa de Desarrollo Productivo Rural (PDPR), una familia de intervenciones que apoyó a 6133 establecimientos familiares pequeños y medianos entre 2012 y 2019 (Durán et al., 2018). A diferencia del programa APA, éste buscaba promover la adopción de un conjunto más amplio de tecnologías, más allá del manejo del agua, y dedicó mayores esfuerzos a la asistencia técnica y la capacitación como instrumentos para la adopción tecnológica. En la evaluación se halló evidencia de que la intervención generó un aumento de la productividad ganadera (de bovinos y ovinos) y lechera, en las dos convocatorias con más cantidad de beneficiarios: el llamado Lechero y el llamado para la Producción Familiar Integral y Sustentable (PFIS); sin embargo, no se identificaron efectos productivos significativos de los llamados Ovino y Agroforestal.

Gracias a la evidencia obtenida sobre los impactos logrados por el PDPR, junto con el análisis del árbol de problemas que se describe más adelante, entre otros factores, el Banco Interamericano de Desarrollo (BID) aprobó el financiamiento de una segunda etapa del Programa y se contribuyó a mejorar el diseño del modelo de intervención. Sin embargo, en un contexto de restricción fiscal, las nuevas autoridades ministeriales decidieron reducir la implementación de varios programas de desarrollo rural. Interesa 
comentar que la evaluación del PDPR permitió conocer que la subpoblación de productores ganaderos y lecheros que fueron beneficiarios por primera vez alcanzaron mayores impactos productivos que aquellos que ya habían recibido apoyos previos, lo que fue considerado por las autoridades como criterio para seleccionar a la población beneficiaria del proyecto Más Agua para el Desarrollo Rural.

Además de evaluar los impactos de las intervenciones sobre los objetivos de largo plazo, también es importante comprender los mecanismos por los cuales se produjeron esos efectos, de modo de mejorar la eficiencia de los esfuerzos públicos a través de un diseño más afinado. Por ese motivo se llevan adelante dos evaluaciones que permitirán conocer si los productores ganaderos beneficiarios del llamado PFIS, ya mencionado, y del programa Ganaderos Familiares y Cambio Climático (GFCC) mejoraron su adopción de tecnologías y prácticas de manejo debido a su participación en la intervención. Adicionalmente, lideradas por AGEV, se llevaron a cabo dos evaluaciones de diseño, implementación y desempeño (DID) que permitieron profundizar el conocimiento de las causas de la problemática y el funcionamiento de las intervenciones (Oficina de Programación y Presupuesto [OPP], 2016; 2017). Según la teoría del cambio del PFIS, el acceso a financiamiento y la asistencia técnica y capacitación permitirían a los productores adoptar mejores prácticas de manejo así como realizar las inversiones requeridas, lo que constituye el mecanismo para alcanzar mayores niveles de productividad. En el caso del proyecto GFCC, el principal objetivo explícito no fue el incremento de la productividad sino reducir la vulnerabilidad de los productores ante la variabilidad del clima. Sin embargo, los mecanismos para lograr una mejor adaptación a la variabilidad climática son, en buena medida, los mismos que los que permiten aumentar la productividad (la adopción de determinadas prácticas de manejo y tecnologías), por lo que se entiende que esta intervención comparte buena parte del árbol de problemas, y el de soluciones, con los otros proyectos que se están evaluando.

Se levantaron las correspondientes encuestas de línea de base, lo que además permitió caracterizar a la población potencial en cada caso (Fuletti, 2017; Gesto et al., 2019). En 2019 y 2020 se realizaron las encuestas de seguimiento, lo que, unido a los registros administrativos del Sistema Nacional de Información Ganadera (SNIG), completa el conjunto de datos para realizar las dos evaluaciones de impacto. Ambas intervenciones están orientadas a poblaciones con similares características y con objetivos también similares, aunque fueron implementadas en distintos momentos y regiones del país y con variantes en las herramientas del programa. A su vez, los datos disponibles para cada evaluación provienen de fuentes diferentes y relevan aspectos complementarios. Así, se espera que los resultados de estas evaluaciones aporten evidencia sobre la efectividad de este tipo de políticas sobre la adopción tecnológica y la mejora productiva, y se generen aprendizajes para mejorar el diseño de futuros programas. 
Adicionalmente, como parte de una tesis de maestría (Baraldo, 2020), se investigó acerca de los impactos productivos del uso de campos de recría (CR) por productores lecheros y los determinantes de su adopción. Un CR es un establecimiento especializado en la fase de recría del ganado (generalmente lechero), gestionado en forma colectiva mediante algún tipo de arreglo asociativo de los productores socios. En teoría, esta estrategia permitiría ganancias de eficiencia por la vía de la especialización: por un lado, permite desarrollar la recría en forma especializada en los CR y, por otro, los predios lecheros pueden también concentrarse en la producción de leche. En la evaluación de impacto no se hallaron efectos significativos del uso de esta tecnología sobre la intensificación productiva de los productores lecheros (aumento de la cantidad de vacas por hectárea), ni sobre la productividad individual por vaca, ni sobre la productividad parcial medida como litros de leche por hectárea lechera.

\section{Evaluaciones costo-beneficio}

Entre 2012 y 2017 la DGDR también ejecutó el proyecto Desarrollo y Adaptación al Cambio Climático (DACC), financiado parcialmente por el Banco Mundial (BM), con el objetivo de mejorar la sustentabilidad económica, social y ambiental de unidades productivas agropecuarias familiares, pequeñas y medianas, a través de la adopción de tecnologías.

Próximo a su finalización, el Área de Evaluación analizó la conveniencia económica de ampliar el proyecto por otros cinco años (DACC adicional). Para ello se realizó un análisis costo-beneficio (ACB) ex ante de la ampliación del proyecto, desde la perspectiva de la sociedad en su conjunto (Aguirre, Baraldo y Durán, 2017). Se consideraron varios escenarios, con los correspondientes análisis de sensibilidad y de riesgos, y se concluyó que la ampliación del proyecto era conveniente económicamente para la sociedad uruguaya y que este resultado era muy poco sensible a variaciones en los parámetros y supuestos del modelo.

Además de los beneficios directos, derivados de una mejora productiva en los establecimientos de los productores y que pueden ser completamente apropiados por los beneficiarios, se consideró otro conjunto de beneficios indirectos que afectan al interés general del sistema en su conjunto. Se destacan en este grupo los cobeneficios ambientales o debidos a la restitución de servicios ecosistémicos, es decir, aquellos bienes, productos o servicios provistos o regulados por los ecosistemas, en beneficio de la humanidad. De modo que se cuantificaron los cobeneficios generados por el restablecimiento del servicio ecosistémico de la calidad del agua en la cuenca del río Santa Lucía (gracias a la mejora del tratamiento de los efluentes en tambos), el secuestro de carbono que permite la gestión inteligente de la ganadería en campo natural y el beneficio implícito 
en la conservación del recurso suelo (gracias a los planes de conservación y uso responsable de suelos agrícolas).

Además, como parte integrante de equipos interinstitucionales, el Área de Evaluación está trabajando en otros tres análisis costo-beneficio.

En cooperación con la Unidad de Gestión de Proyectos (UGP) del MGAP se desarrolla un análisis de factibilidad sobre la creación de un distrito de riego en Colonia Valdense. Uno de los aportes más interesantes es la incorporación explícita de la variabilidad climática, mediante una distribución aleatoria que afecta al mismo tiempo a los costos operativos (a través de la demanda de riego) y al beneficio incremental del proyecto (a través del rendimiento agrícola de secano, o sin proyecto). A partir del escenario base se realizaron simulaciones Montecarlo para determinar el riesgo de que el VAN (valor actual neto, indicador de conveniencia financiera del proyecto) fuese negativo. Se realizaron ejercicios que tomaban en cuenta las perspectivas de los principales agentes: el operador del sistema y los productores usuarios; esta perspectiva es más rica en cuanto permite que surjan resultados que dependen de interacciones estratégicas entre los actores y que quedarían ocultos en un análisis agregado.

En segundo lugar, el MGAP junto con el Instituto Nacional de Investigación Agropecuaria (INIA) y el Instituto Nacional de Carnes (INAC) encomendaron a un equipo de consultores internacionales una evaluación sobre los impactos económicos y sociales de un cambio en el estatus de prevención y control de la fiebre aftosa en Uruguay y sobre las implicaciones de los riesgos asociados. El Área de Evaluación participó como contraparte en diversos talleres técnicos, incluido un análisis costo-beneficio.

Finalmente, el Área de Evaluación forma parte del grupo de trabajo ejecutivo creado por el ministro para elaborar un plan estratégico para el control/erradicación de la Cochliomyia hominivorax (mosca de la bichera o gusano barrenador del ganado). La Organización Internacional de Energía Atómica (OIEA) propuso un plan sanitario basado en la técnica del insecto estéril (TIE) (Vargas Terán y Ortiz, 2019). El trabajo de evaluación ex ante incluyó el diseño e implementación de una encuesta autoadministrada a productores sobre diversos aspectos productivos y económicos vinculados a la bichera y una consultoría externa que realizó un análisis costo-beneficio, en la que el Área participó como contraparte nacional (Köbrich, 2019). Se concluye que la estrategia de control y erradicación propuesta es conveniente desde el punto de vista de la economía uruguaya en su conjunto. 


\section{Evaluaciones con modelos de equilibrio general computable}

El Área de Evaluación trabaja con un modelo de equilibrio general computable (MECG) que permite analizar los efectos de diversas políticas y shocks exógenos sobre un conjunto amplio de variables macroeconómicas (PIB, exportaciones, importaciones, tipo de cambio real, recaudación tributaria, etc), así como sobre indicadores de desempeño sectorial (VAB, exportaciones, empleo, de los principales sectores de actividad económica del país).

El MEGC se está aplicando en la evaluación económica del programa sanitario para la erradicación de la bichera desde el punto de vista de la sociedad en su conjunto, tomando en cuenta tanto los efectos directos como los indirectos o derrames de la política pública. El MEGC permite, además, analizar el impacto de distintas formas de financiamiento de la campaña sanitaria.

\section{Evaluaciones con metodologías cualitativas}

Como se adelantó, además de aplicar técnicas cuantitativas, el Área de Evaluación ha desarrollado evaluaciones y contribuciones basadas en técnicas cualitativas En la evaluación de resultados del Programa de Fortalecimiento Institucional (PFI) (Artía et al., 2017; Durán et al., 2016), un equipo multidisciplinario realizó entrevistas individuales y grupales a organizaciones rurales participantes del llamado y recogió una percepción mayoritariamente favorable de un amplio grupo de directivos, técnicos y socios de organizaciones participantes. Entre los principales logros alcanzados por la intervención se destacan las mejoras en los procesos organizativos internos a las instituciones beneficiarias, aunque con menores avances en la cantidad y calidad de los servicios que brindan esas organizaciones a los productores, así como en la dimensión de manejo sostenible de los recursos naturales.

Por su parte, la elaboración del árbol de problemas que fundamenta la teoría del cambio del PDPR y del proyecto DACC fue un trabajo conjunto entre el Área de Evaluación y la DGDR (Aguirre et al., 2018). Se identificó que el principal problema que enfrentan las unidades de producción familiares, pequeñas y medianas es la baja sostenibilidad económica, social y ambiental. Se analizaron las causas y consecuencias de dicho problema y se identificó la teoría del cambio de los programas que se implementan para contribuir a solucionar el problema. La principal contribución de este producto de evaluación es la mejora en el diseño de las próximas intervenciones, al tiempo que será la 
base para la evaluación de impacto de diversos programas y proyectos dirigidos a productores familiares, pequeños y medianos.

El Área se desempeñó como contraparte técnica en una consultoría para el análisis de los institutos públicos no estatales del sector agropecuario de Uruguay (Arboleya, 2019). Ello implicó la participación en entrevistas a presidentes y gerentes de los institutos, y a directores de unidades ejecutoras del MGAP. Tomando como base un estudio anterior (Ministerio de Ganadería Agricultura y Pesca [MGAP] y Centro Cooperativista Uruguayo [CCU], 2007), la consultoría se orientó a actualizar el análisis del estado de los institutos vinculados al MGAP. Se señalan los principales cambios observados en la última década, particularmente un impulso a la incorporación de nuevas prácticas de monitoreo y evaluación de resultados en la institucionalidad agropecuaria, aunque con heterogeneidad entre los institutos. Del estudio surgen dos recomendaciones principales: 1) consolidar las instancias de trabajo conjunto que ya tienen un cierto grado de institucionalización (ej.: gabinetes y consejos), incorporando el diálogo sobre experiencias y prácticas de gestión; 2) reforzar las capacidades ministeriales de monitoreo y evaluación de las planificaciones y los resultados de las políticas e instrumentos ejecutados por los institutos.

Finalmente, cabe mencionar otros estudios que el Área de Evaluación realiza y que contribuyen con las evaluaciones. Así, se desarrolló e implementó una metodología de cálculo, a nivel microeconómico, de la producción de carne y medidas parciales de la productividad ganadera (Aguirre, 2018). Estimar la productividad cárnica a nivel micro es una necesidad para evaluar el impacto de políticas ganaderas y monitorear el desarrollo de la cadena cárnica. Estos datos han sido utilizados para evaluar el impacto de programas de mejora productiva en la ganadería de carne (Durán et al., 2018), para realizar análisis descriptivos (Aguirre, 2018; 2019) y para construir modelos estadísticos para predecir la productividad.

\section{Comentarios finales}

La evaluación de políticas públicas se puede concebir como un proceso de aprendizaje institucional del que surgen insumos para mejorar el diseño y la implementación de las políticas. Ayuda a mejorar la efectividad del gasto público, al determinar si los programas y políticas públicas son buenas inversiones sociales y si logran contribuir a los objetivos propuestos. Este proceso también contribuye a legitimar y transparentar el ciclo de política pública y favorece la rendición de cuentas. Por lo tanto, evaluar es importante para mejorar las intervenciones y para generar criterios de asignación del gasto público. 
El desafío de la evaluación es producir información útil y pertinente que responda a las necesidades y demandas de actores diversos (técnicos, políticos y la ciudadanía) y asegure rigurosidad de acuerdo con estándares científicos.

La política pública del MGAP, objeto de estudio del Área de Evaluación, se estructura en torno a un conjunto de líneas estratégicas (inserción internacional competitiva, intensificación sostenible, adaptación al cambio climático, desarrollo rural, articulación institucional, conciencia agropecuaria), que contribuyen y se alinean con los Objetivos de Desarrollo Sostenible al 2030 de las Naciones Unidas. De esta manera, la evaluación de las políticas agropecuarias contribuye también al logro de las metas de la agenda del desarrollo sostenible, aportando evidencia y análisis para redirigir los esfuerzos y mejorar el diseño de las intervenciones de política.

En los últimos años, los avances en el Área de Evaluación de Políticas Agropecuarias contribuyeron a fortalecer la cultura de evaluación en el MGAP a través de: 1) conformación de un equipo técnico especializado que articula con las diversas unidades ministeriales; 2) fortalecimiento de capacidades para evaluar políticas con distintas técnicas y metodologías; 3) creación de un sistema de información que aprovecha la información existente y la consolida con encuestas; 4) retroalimentación mediante la participación de actores internos y externos durante el proceso de evaluación.

Aunque todavía hay mucho por avanzar, las autoridades ministeriales de sucesivas administraciones de gobierno se han ido apropiando del proceso de evaluación y de sus frutos. Esto incluye a varios ministros, subsecretarios y directores del MGAP que incorporaron resultados de las evaluaciones en sus discursos y tomaron en cuenta algunas de las recomendaciones de evaluación en la formulación y rediseño de intervenciones.

Para finalizar, interesa destacar que el aporte del proceso de evaluación a la mejora de las políticas excede a la mera publicación de los resultados de las evaluaciones. En efecto, más allá de los informes finales, tanto los tomadores de las decisiones de política como los implementadores se benefician de su participación en los debates y análisis colectivos que se realizan durante el proceso de evaluación, lo que constituye un aporte sumamente valioso y de utilidad para la mejora de las herramientas e intervenciones y orienta las decisiones hacia el logro de los objetivos.

\section{Referencias bibliográficas}

Aguirre, E. (2018). Evolución reciente de la productividad ganadera en Uruguay (20102017): Metodología y primeros resultados. Anuario OPYPA, 457-470.

Aguirre, E. (2019). Productividad ganadera de los establecimientos de carne bovina del Censo General Agropecuario. Anuario OPYPA, 425-439. 
Aguirre, E., Baraldo, J., y Durán, V. (2017). Evaluación costo beneficio ex-ante del Proyecto DACC Adicional. Anuario OPYPA, 521-542.

Aguirre, E., Baraldo, J., Durán, V., Fuletti D., Hernández, E., Mila, F., y Olascuaga, J. (2018). Problemas a abordar por parte de la Dirección General de Desarrollo Rural (DGDR). Anuario OPYPA, 399-408.

Aguirre, E., Baraldo, J., Durán, V., Gesto N., Ott, F., y Móttola, J. P. (2017). Evaluación del impacto del proyecto Agua para la Producción Animal en la productividad de productores lecheros. Anuario OPYPA, 457-470.

Arboleya, I. (2019). Consultoría en análisis de los institutos públicos no estatales del sector agropecuario de Uruguay: Informe final de consultoría BID. (Manuscrito inédito).

Artía, P., Bazzi, C., y Durán, V. (2017). Resultados preliminares de evaluación del programa de fortalecimiento institucional para el desarrollo rural sustentable (PFI). Anuario OPYPA, 505-519.

Baraldo, J. (2020). Campos de recría lecheros en Uruguay: determinantes de la adopción y efectos causales. (Tesis de Maestría en Economía). Facultad de Ciencias Económicas y Administración, Universidad de la República, Montevideo.

De Janvry, A., Sadoulet, E., y Suri, T. (2017). Field experiments in developing country agriculture. En A. Vinayak Banerjee y E. Duflo (eds.), Handbook of economic field experiments, 2, 427-466. North-Holland: Elsevier.

Departamento Nacional de la Planeación. (2012). Guía para la evaluación de políticas públicas. Recuperado de https://politicaspublicasydeporte.files.wordpress.com/ 2018/09/guc3ada-para-la-evaluacic3b3n-de-pp.pdf.

Durán, V., Ackermann, M. N., Baraldo, J., y Mila, F. (2014). La estrategia del MGAP para la evaluación de políticas agropecuarias. Anuario OPYPA, 269-280.

Durán, V., Aguirre, E., Artía, P., Baraldo, J., Fuletti, D. y Hernández, E. (2016). Avances en la evaluación de políticas agropecuarias en el MGAP. Anuario OPYPA, 283-314.

Durán, V., Aguirre, E., Baraldo, J., Fuletti, D. y Hernández, E. (2018). Primera evaluación del Programa de Desarrollo Productivo Rural. Anuario OPYPA, 583-597.

Durán, V.; Aguirre, E., Baraldo, J., Hernández, E., y Laguna, H. (2019). Cinco años de consolidación de la evaluación de políticas agropecuarias en el MGAP. Anuario OPYPA, 425-439.

Durán, V., y Hernández, E. (2019). Guía para la evaluación de políticas agropecuarias en el MGAP. Anuario OPYPA, 371-376.

Fuletti, D. (2017). Informe de línea de base del programa ganaderos familiares y cambio climático. Anuario OPYPA, 461-473.

Gesto, N., Lado, A., y Laguna, H. (2019). informe de caracterización de la población potencial ganadera del programa producción familiar integral y sustentable. Anuario OPYPA, 583-596. 
HM Treasury. (2020). Magenta book: Central government guidance on evaluation. Recuperado de https://assets.publishing.service.gov.uk/government/uploads/system/uploads/attachment_data/file/879438/HMT_Magenta_Book.pdf.

Independent Evaluation Group. (2011). Impact evaluations in agriculture: An assessment of the evidence. Washington: World Bank.

Köbrich, G. (2019). Estudio de factibilidad económica para la erradicación progresiva del gusano barrenador del ganado de Sudamérica utilizando la técnica del insecto estéril: Etapa inicial - Uruguay y sus zonas fronterizas con Argentina y Brasil. OIEA.

López, C., Salazar, L., y De Salvo, C. (2017). Gasto público, evaluaciones de impacto y productividad agrícola: Resumen de evidencias de América Latina y el Caribe. Recuperado de http://dx.doi.org/10.18235/0000627.

López, F., y Maffioli, A. (2008). Technology adoption, productivity and specialization of uruguayan breeders: Evidence from an impact evaluation. Recuperado de https:// publications.iadb.org/publications/english/document/Technology-AdoptionProductivity-and-Specialization-of-Uruguayan-Breeders-Evidence-from-an-Impact-Evaluation.pdf.

Ministerio de Ganadería, Agricultura y Pesca y Centro Cooperativo Uruguayo. (2007). Análisis de los Institutos Públicos: Una agenda de temas para la Institucionalidad Pública Agropecuaria. (Manuscrito inédito)

Mullally, C., y Maffioli, A. (2016). Extension and matching grants for improved management: An evaluation of the uruguayan livestock program. American Journal of Agricultural Economics, 98(1), 333-350.

Oficina de Programación y Presupuesto. (2016). Evaluación DID: Ganaderos Familiares y Cambio Climático (GFCC). Recuperado de https://transparenciapresupuestaria.opp.gub.uy/inicio/registro-nacional-de-evaluaciones/evaluaci\%C3\%B3ndid-ganaderos-familiares-y-cambio-clim\%C3\%A1tico-gfcc.

Oficina de Programación y Presupuesto. (2017). Evaluación DID: Producción Familiar Integral y Sustentable (PFIS). Recuperado de https://transparenciapresupuestaria.opp.gub.uy/inicio/registro-nacional-de-evaluaciones/evaluaci\%C3\%B3ndid-producci\%C3\%B3n-familiar-integral-y-sustentable-pfis.

Vargas Terán, M., y Ortiz, G. (2019). Propuesta de un Plan Estratégico Subregional para la Erradicación del Gusano Barrenador del Ganado C hominivorax en América del Sur: Proyecto fortalecimiento de las capacidades para la evaluación de la factibilidad de un programa de control progresivo del GBG OIEA/RLA/5/075. (Manuscrito inédito). 